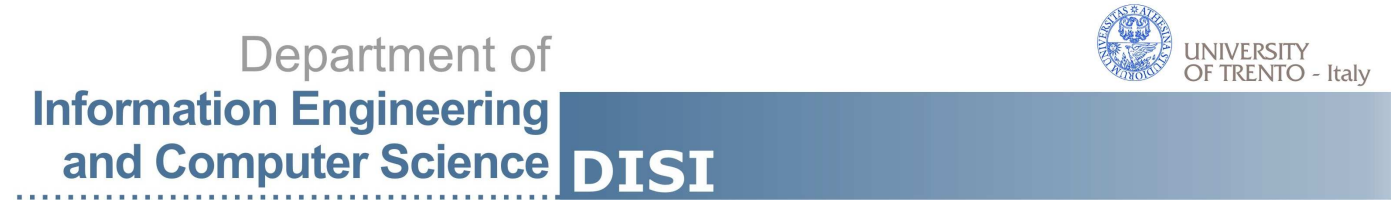

DISI - Via Sommarive, 5 - 38123 POVO, Trento - Italy

http://disi.unitn.it

\title{
TBIM: A LANGUAGE FOR MODELING AND REASONING ABOUT BUSINESS PLANS.
}

Fabiano Francesconi, Fabiano Dalpiaz and John Mylopoulos.

May 2013

Technical Report \# DISI-13-020 



\title{
TBIM: a Language for Modeling and Reasoning about Business Plans
}

\author{
Fabiano Francesconi ${ }^{1}$, Fabiano Dalpiaz ${ }^{2}$, and John Mylopoulos ${ }^{1}$ \\ 1 University of Trento, Italy - \{francesconi, jm\}edisi.unitn.it \\ ${ }^{2}$ University of Toronto, Canada-dalpiazecs.toronto.edu
}

\begin{abstract}
Conceptual models of different aspects of an organizationbusiness objectives, processes, rules, policies and objects-have been used for organizational design, analysis, planning, and knowledge management. Such models have also served as starting points for designing information systems and conducting business intelligence activities. This paper proposes the Tactical Business Intelligence Model (TBIM), a language for modeling and reasoning about strategic business plans. TBIM lies in between the strategic and tactical level, for strategic plans are abstract tactics. The language extends a strategic modeling language with primitives for business model design. The paper presents graphical syntax and semantics for TBIM, and illustrates the power of our proposal on a medium-sized case study. In addition, the paper proposes a method for evaluating alternative strategic plans by mapping them to business process models and analyzing the outcome via simulation techniques.
\end{abstract}

Keywords: strategic planning; organizational models; business models

\section{Introduction}

Organizations rely on a hierarchy of management layers, each focusing on different aspects of the organization. Conceiving an organization in terms of layers eases decision-making and management activities, for it refines the task at hand into smaller tasks at different levels of abstraction.

The topmost management level, called strategic, defines the direction of the organization. Notions such as vision, mission, and goal are essential components of a strategy. Once the strategy is set, a crucial decision is to be taken: how does the organization realize it? This question is answered by conducting strategic planning [113 activities, which lead to the definition of a high-level business tactic that, if implemented correctly, is expected to realize the strategy.

Strategic planning success depends on many factors, including the expertise of the management, the adoption of best practices, and the analysis of the key aspects of a business tactic (choosing the right ontology). While the topic has been widely explored in management science, there has been little work grounded on the usage of conceptual models to represent and analyze strategic plans.

In this paper, we propose the Tactical Business Intelligence Model (TBIM), a language for modeling and reasoning about strategic plans. The language is a 
link between the strategic level and the tactical level in the sense that a business plan comprises a set of business goals, as well as the tactical plans for reaching those goals, defined in terms of value propositions, market segments, distribution channels, production and delivery activities, as well as partnerships. TBIM builds on and extends state-of-the-art modeling techniques: (i) the Business Intelligence Model [12, a strategic modeling language based on primitives such as goal, situation, indicator, and (ii) the Business Model Ontology [19, which offers a core set of concepts to conceive business models at a tactical level.

Specifically, the contributions of the paper are as follows:

- We introduce syntax and semantics for the TBIM modeling language. TBIM acts as a bridge between strategic and tactical models.

- We define a graphical notation for TBIM. The notation consists of two complementary views: the tactical view focuses on the internal aspects of a tactic, while the partnership view models the partnerships among enterprises.

- We provide a method for comparing alternative TBIM tactics through business process analysis and simulation techniques. Our method helps refining abstract TBIM tactics into more detailed tactics expressed as BPMN models.

- We illustrate our approach through snippets from a medium-sized case study concerning the organization of an international jazz festival [19].

Organization. Section 2 reviews our baseline. Section 3 introduces syntax and semantics of TBIM. Section 4 presents OCL syntactic constraints for BIM and TBIM. Section 5 defines how TBIM models can be mapped to BPMN models. Section 6 illustrates how BPMN analysis techniques are useful to evaluate alternative TBIM tactics. Section 7 discusses related work, while Section 8 concludes.

\section{Baseline}

Our baseline consists of BIM, a modeling language for strategic business modeling (Section 2.1), and a business ontology that defines the key factors to model a business tactic (Section 2.2). Our aim is to combine the set of modeling primitives provide by these approaches into a modeling language for strategic planning.

\subsection{Business Intelligence Model (BIM)}

The Business Intelligence Model (BIM) [12 is a modeling language for representing business strategies. BIM relies on primitives that decision makers are familiar with, such as goal, task/process, indicator, situation, and influence relations. BIM supports the notions from SWOT (Strengths, Weaknesses, Opportunities, Threats) analysis [4 by modeling internal and externals factors (situations) that are (un)favorable for fulfilling certain goals. BIM comes with automated reasoning techniques, including "what if?" and "is it possible?" analyses [12.

Figure 1 briefly illustrates the syntax of BIM by modeling part of the Montreaux Jazz Festival (MJF) organization case study [19]. 


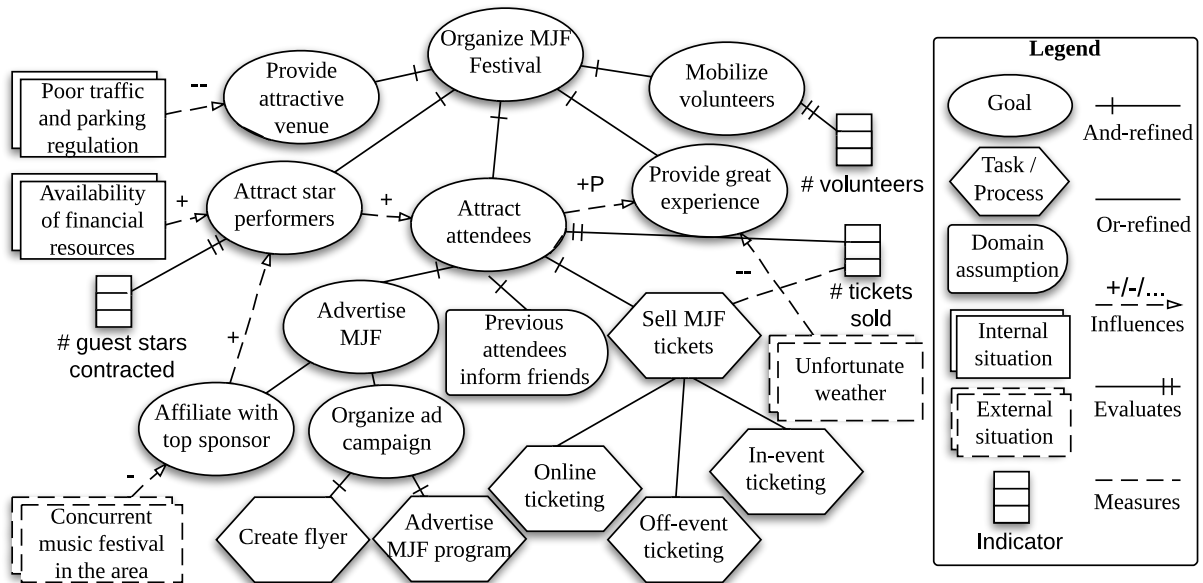

Fig. 1: Partial BIM model for the Montreaux Jazz Festival case study

The top-level strategic goal is to Organize MJF Festival. To achieve this goal, five subgoals are to be pursued and fulfilled, including Provide attractive venue, Attract star performers, and Attract attendance. The latter goal requires to achieve goal Advertise MJF and to execute task Sell MJF tickets, assumed that Previous attendees inform their friends. The festival can be advertised by either affiliating with a top sponsor who also takes care of advertising, or internally organizing an ad campaign (which requires processes Create flyer and Advertise MJF Program). Goals and situations influence one another. The external situation Other music festival in the area influences negatively goal Affiliate with top sponsor, while the internal situation Availability of financial resources positively influences goal Attract star performers. Indicators are associated with goals so to evaluate to what extent the goal is fulfilled. For example, \# volunteers evaluates goal Mobilize volunteers.

\subsection{Business Model Ontology (BMO)}

The Business Model Ontology (BMO) [19] argues for a set of success factors for e-business organizations. BMO is centred on four pillars (the dashed-border rectangles in Figure 2]:

- Product innovation is achieved when the company defines a value proposition that effectively reaches one or more customer segments by offering novelty, lower prices, or customer relationship excellence.

- Infrastructure management describes the value system configuration to deliver the value proposition, which includes defining partnerships and carrying out activities which use, consume, and produce resources.

- Customer relationship needs establishing high-quality client relationships, and reaching different client segments via adequate distribution channels. 
- Financial aspect is a cross-cutting concern in every organization. Defining a right balance between the revenue model and the cost structure is essential for the survival of the organization in the market.

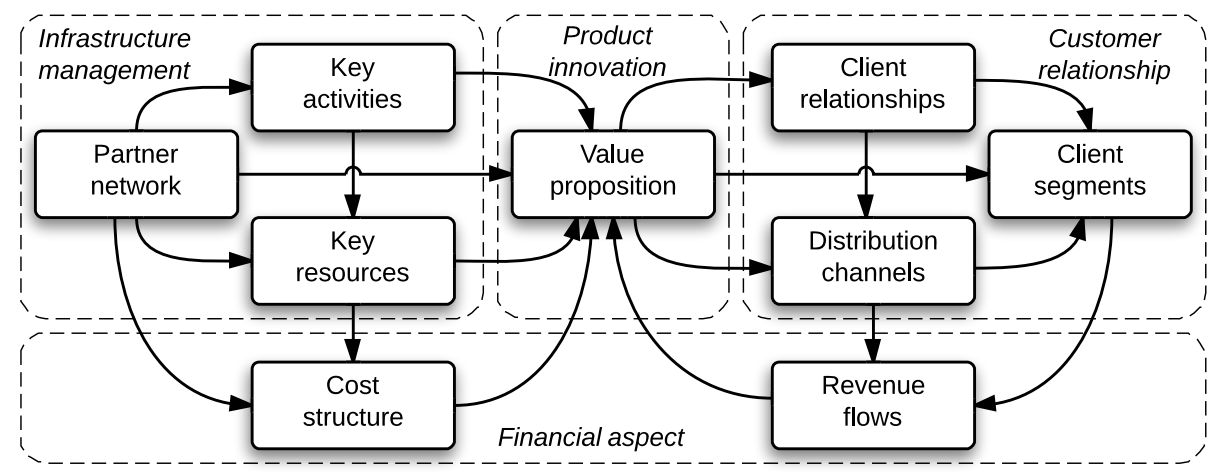

Fig. 2: Outline of the Business Model ontology

\section{Tactical Business Intelligence Model (TBIM)}

We present the metamodel and the graphical syntax of TBIM. TBIM combines the strategic modeling framework provided by BIM with key elements from BMO. Our analysis of BMO led us to the following set of requirements for extending BIM towards strategic planning:

R1. Market segments. Products and services are typically made available to specific customer segments. The language should be able to define what products and services an organization offers and to whom.

R2. Cross-organizational relationships. The success of a strategic plan heavily depends on the establishment and maintenance of a network of partnerships with other organizations.

R3. Distribution channels. Products and services are distributed through different channels. The choice of a specific channel depends on the customer segment that is approached by the provider.

R4. Resources and value propositions. In order to create value, organizations use, create, consume, and transform resources [25]. Value propositions are resources that are a source of revenue for an organization [19].

TBIM consists of two complementary modeling views. The tactical view (Section 3.1 uses an extended version of BIM to describe the strategy of the modeled organizations as well as the high-level tactic to fulfill their goals. The partnership view (Section 3.2 represents a network of contractually-related organizations. Together, these two views do model alternative business plans (Section 3.3). 


\subsection{Tactical View}

The UML class diagram in Figure 3 presents the metamodel of the tactical view. The gray-colored classes are adopted from BIM. We illustrate the graphical notation through the TBIM tactical view diagram in Figure 4.

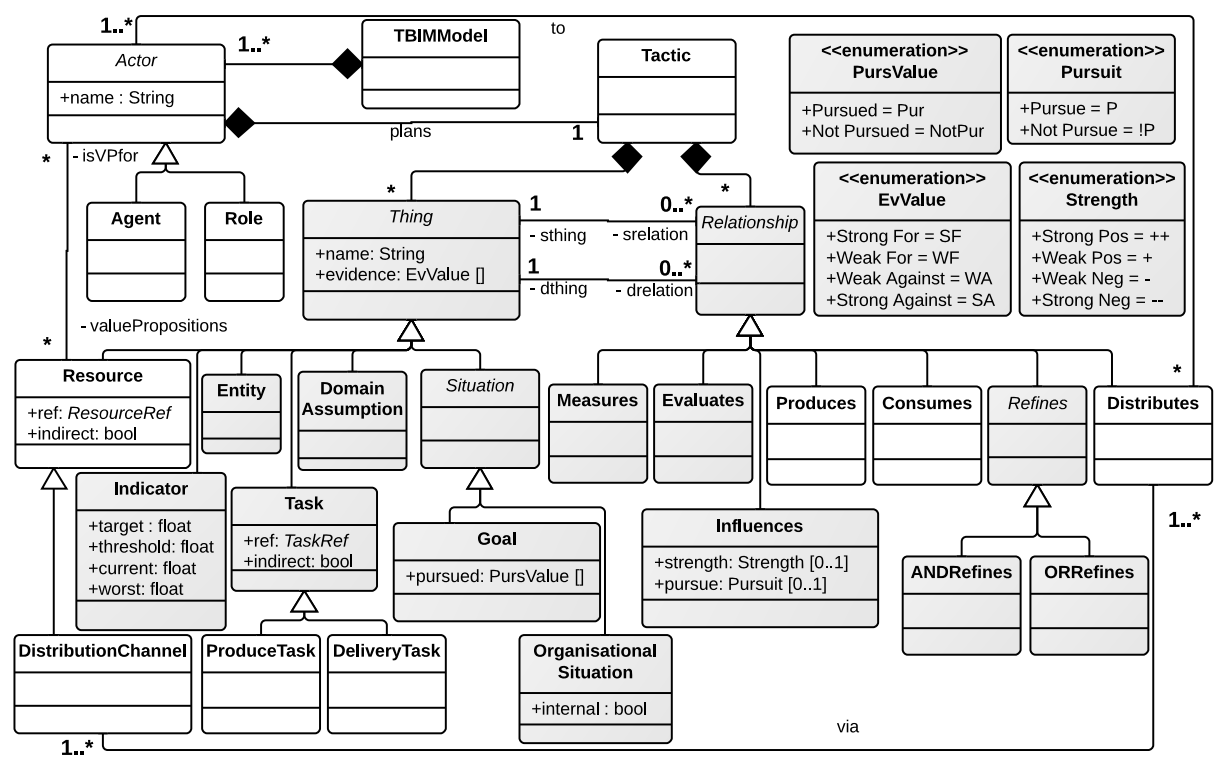

Fig. 3: Metamodel of the tactical view. Classes in gray are adopted from BIM

Agent and role. Agents represent a concrete organization or person. An agent is an active entity that carries out actions to achieve goals by exercising its knowhow 28. Agents are intentional, for they carry out activities to achieve their goals. Roles are an abstract characterization of the behavior of a social agent within some specialized context or domain of endeavor. The term Actor refers generically to an agent or a role (is-a relationship in Figure 3). In Figure 4 , for example, MJF is an agent that represents the festival organizers, while Local customer, Loyal customer, etc. are roles representing different types of customer.

Unlike BIM, TBIM models consist of multiple actors. Consequently, BIM entities such as goals and tasks fall within the scope of a specific actor.

Resource and value proposition. Resources are anything of value for the company being modeled. A resource can be animate (e.g., human, animal, etc.) or inanimate (e.g., wood, chair, money, etc.). In Figure 4 , Blank papers are resources for the agent MJF. TBIM also includes value propositions as a specialization of resources. A value proposition is the statement of benefits that are delivered by the firm to its external constituencies [3]. They differ from plain resources as they carry an intrinsic value for the company, and they form its primary source 


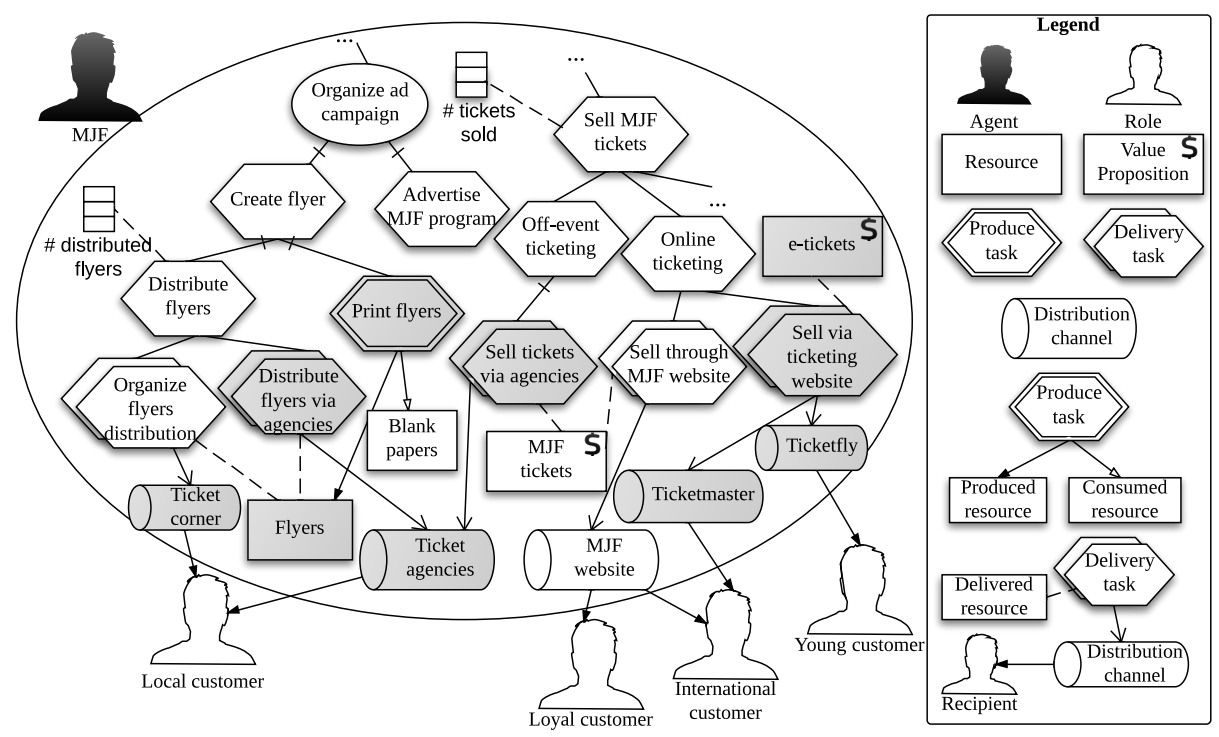

Fig. 4: Partial TBIM tactical view for the MJF case study. Gray-colored elements are indirect (obtained from other actors)

of revenue. For instance, MJF tickets are a value proposition for MJF, because their sales produce revenue for the festival. On the contrary, Flyers are a resource that does not carry revenue directly. TBIM distinguishes between direct entities (resources, tasks, distribution channels) that are originally part of an actor, and indirect entities that are acquired from others via partnerships (see Section 3.2). For example, Flyers are an indirect resource, meaning that MJF obtains them from another actor. In our graphical notation, indirect entities are gray-colored.

Produce task, produces, and consumes. A Produce Task is a set of activities that results in the production of resources. A produce task is linked to produced resources via Produces relationships, and it can be connected to some resources via Consumes relationships, to indicate that the production process consumes those resources. Produce tasks specialize BIM tasks. While BIM tasks can be decomposed into produce tasks, the latter type of tasks can not be decomposed, for their semantics is already very specific. In order to express that multiple produce tasks are needed, one can decompose a generic task into multiple produce tasks, each connected to an individual resource. In Figure 4. Print flyers is a produce task that consumes resource Blank papers and produces resource Flyers.

Distribution channel and delivery task. Distribution channels are means through which customers are delivered resources. Delivery tasks are tasks indicating that resources are distributed to other actors. These tasks include the whole process of distributing a product, including packaging, shipment scheduling, and delivery. A delivery task is connected to at least one distribution channel. Multiple distribution channels can be associated with a delivery task to reach 
different market segments. Delivery tasks can not be further refined, but they can be refinements of a generic task. In Figure 4, Sell through MJF website is a delivery task, which encompasses the delivery of the value proposition MJF tickets through the channel MJF website to two types of customers: Loyal and International.

\subsection{Partnership View}

Establishing and maintaining a network of partnerships is key to the success of a company [5]10]19]24, and contributes to its competitiveness. In TBIM, partnerships enable fulfilling strategic plans. Partnerships are stipulated through contractual agreements that specify which artifacts are made available, to whom, and in exchange for what. TBIM supports partnerships modeling through the partnership view. Its metamodel is in Figure 5 and illustrated in Figure 6.

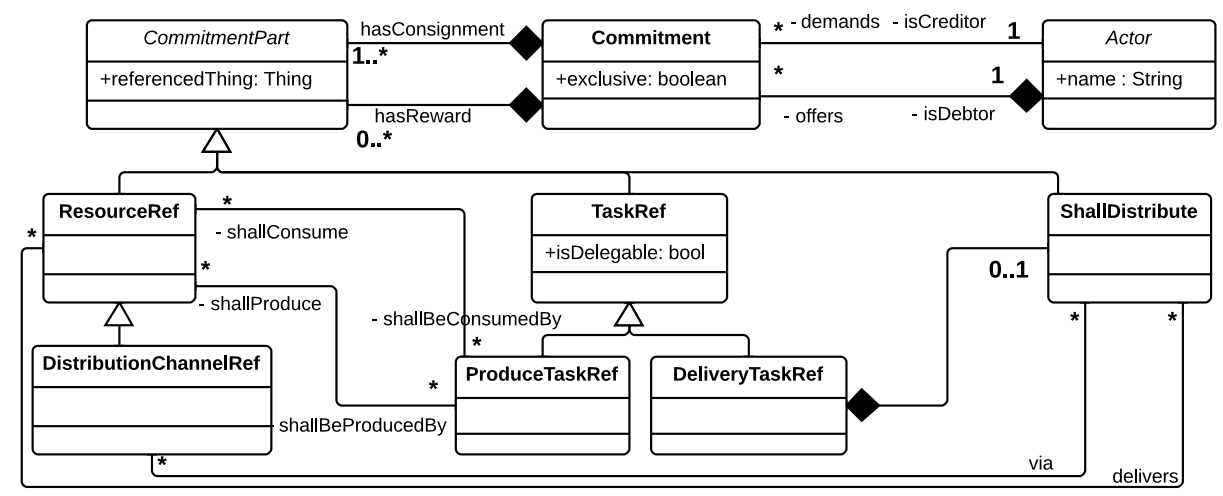

Fig. 5: Metamodel of the partnership view

Commitment. Commitments are the core element of the partnership view. A commitment is a contractual agreement among actors on the execution of tasks, exchange of resources, and provision of distribution channels. It abstracts away operational details [22, minimally constraining business executions. A commitment is a quaternary relation where a debtor actor commits to a creditor actor that a consignment will be delivered/provided, if (optionally) a reward is provided by the creditor actor.

Commitments relate elements that appear in the tactical view: debtor and creditor are chosen among agents and roles, while resources, tasks (of all types), and distribution channels constitute the consignment and the reward. Since resources, tasks, and distribution channels appear (are contained in the tactical view within the scope of an actor, the metamodel of the partnership view includes references to those objects: ResourceRef, TaskRef, and DistributionChannelRef. The consignment and reward indicate the commitment of the involved actors to:

- Resource provision: a resource shall be transferred. 


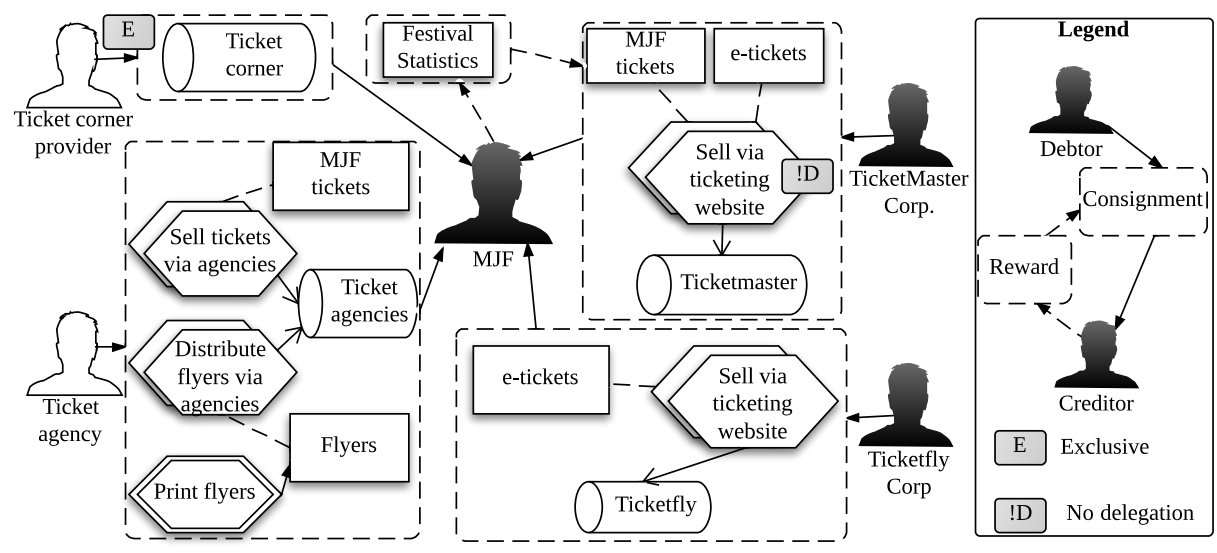

Fig. 6: Partial TBIM partnership view for the MJF case study

- Task execution: a generic process/task shall be carried out.

- Produce task execution: a production line is provided for producing resources.

- Delivery task execution: a delivery service for some items is made available.

- Distribution channel provision: a distribution channel is provided to enable a distribution process.

In Figure 6, role Ticket agency commits to agent MJF to execute produce task Print flyers (to produce Flyers), and delivery tasks Distribute flyers via agencies and Sell tickets via agencies. For both deliveries, the channel Ticket agencies will be used. The commitment from TicketMaster Corp. to MJF shows rewards: tickets are sold via the Ticketmaster channel only if Festival Statistics are provided from MJF.

A commitment defining a partnership can be further constrained:

- ShallDistribute (Figure 5) indicates that a delivery task shall deliver a specified set of resources via a specified set of distribution channels.

- A commitment may be exclusive, meaning that the consignment shall be provided to the creditor only. The commitment from Ticket corner provider is exclusive: the Ticket corner shall be used for selling MJF tickets only.

- A task reference in a commitment can be delegable (default) or not. If delegable, the debtor is authorized to delegate task execution to another actor. TicketMaster Corp. commits to not delegate task Sell via ticketing website.

\subsection{Business plans}

The tactical and the partnership view of TBIM are used to represent alternative business plans to achieve the strategic business goals in the considered domain.

Business plan. It is a formal statement of a set of business goals, and the plan for reaching those goals, defined in terms of value propositions, market segments, distribution channels, production and delivery activities, as well as partnerships. 
Figures 4 and 6 include alternative business plans. Goal Organize ad campaign requires executing tasks Create flyer and Advertise MJF program. The former task requires the indirect production task Print flyers, which consumes Blank papers and produces Flyers, and is supported by the commitment from Ticket agency in the partnership view (Figure 6).

To distribute flyers, alternatives exist: either flyers distribution is organized internally, or the delivery task Distribute flyers via agencies is chosen. The latter solution distributes Flyers to the market segment of Local customers through channel Ticket agencies. The commitment from Ticket agency supports this plan.

\section{Syntactic constraints}

The metamodels illustrated in Figure 3 and Figure 5 are over-expressive with respect to our intended semantics. The set of possible relationships between each Thing element needs to be narrowed down. In the following sections, we formalize the allowed syntax of the BIM and TBIM languages using the OCL language [18. Section 4.1 presents the rules for constraining the BIM language, whilst Section 4.2 shows the rules for the TBIM language. Since TBIM is an extension of the BIM language, the rules presented in the next section apply for TBIM as well.

\subsection{BIM syntactic constraints}

General constraints. The constraint presented in OCL Rule 1.1 disallows reflexive relationships, where an element is related to itself.

OCL Rule 1.1: No self-referencing

context Relationship

inv NoSelfReference: self.sthing<>self.dthing

Recall that OCL Rules are inherited by elements subclassing an existing constrained element. Thereof, all the relationships in the BIM/TBIM ecosystem are going to be supervised by the aforementioned rule.

Refines. The refines relationship allows business elements to be refined so to achieve lower levels of granularity. Specifically, the refines relationship works only among elements sharing the same type: a situation can be refined into other situations, an indicator into other indicators, a task into other tasks. Yet, goals make an exception since those can be refined into subgoals as well as subtasks. OCL Rule 1.2 enforces this behavior:

OCL Rule 1.2: Refines constraints

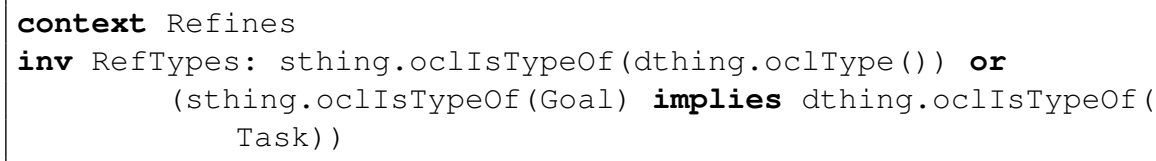


Another OCL Rule is provided in 1.3 and enforces the non-existence of cycles of refines relationships. The constraint (backwardly) evaluates all the refines relationship in a diagram and it evaluates to false if there exists a refines relationship path from an element to itself. Intuitively, the semantics of refines suggests that the refiner is supposed to be more specific than the refined; therefore, it does not make sense to have circularities in this relationship.

OCL Rule 1.3: Refines non-circularity constraint

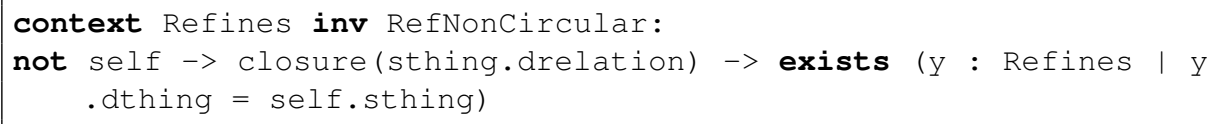

AND/OR-Refines. These relations allow elements to be refined into an AND/OR fashion, accordingly. Recall that refines is a binary relationship. Therefore, the decomposition of an element into others shall be expressed using a set of refines relationships. All of these are expected to share the same type (AND/OR).

OCL Rule 1.4: Refines logical constraints

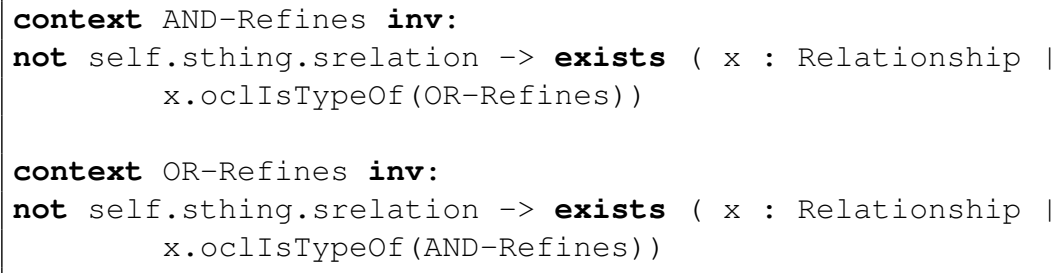

The OCL Rules 1.4 for AND/OR-refinement impose that the source element has not been OR-Refined in the model, if it has already been AND-Refined. The converse applies as well for the OR-Refines relationship.

Influences. The influences relationship is used to represent the transmission of (un)favorable effects on situations (i.e. goals and organizational situations). This relationship only applies from situations to to situations; OCL Rule 1.5 enforces that.

OCL Rule 1.5: Influences constraint

context Influences inv:

sthing.oclIskindof(Situation) and dthing.ocliskindof(Situation)

Evaluates. Indicators evaluate situations (and hence goals). OCL Rule 1.6 enforces the source to be an indicator and the target to be a situation.

OCL Rule 1.6: Evaluates constraint

context Evaluates inv:

sthing.oclistypeof(Indicator) and dthing.ocliskindof(Situation)

Measures. Indicators can be associated with a particular task via the measures relationship. The measure link is intended to be an abstract relationship that 
associates a particular indicator with the task that it measures. The OCL constraint 1.7 enforces the source element to be an indicator and the destination to be a task.

OCL Rule 1.7: Measures constraint

context Measures inv:

sthing.oclistypeof(Indicator) and dthing.oclistypeof(Task)

\subsection{TBIM syntactic constraints}

In the previous section we provided the OCL Rules for the BIM language. These rules serve as baseline for those we present in this section. Given that TBIM is built on top of the BIM language, it also inherits the rules for the concepts sharing the same super-type.

Refines. The OCL Rule 1.2 enforces the refines relationship to be applied between elements sharing the same type, with the sole exception for goals. We extend such specifications in OCL Rule 1.8 by adding new constraints for TBIM elements. Specifically, it enforces also the following: (a) resources cannot be refined; (b) tasks can be refined into delivery task or produce task; (c) produce task cannot be refined; (d) delivery task cannot be refined.

OCL Rule 1.8: Refines constraints (extension of BIM's)

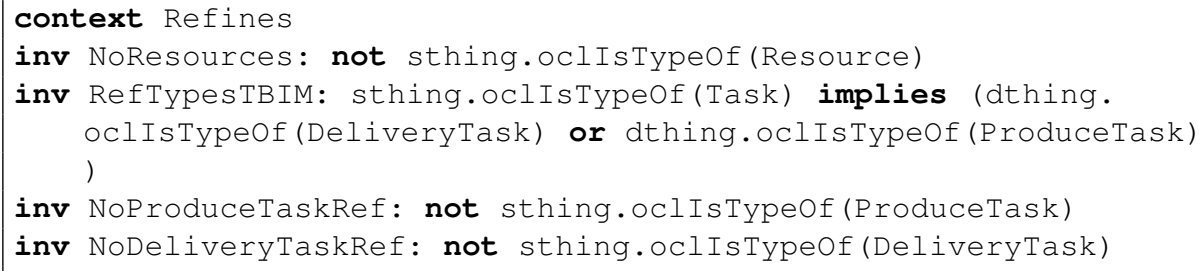

Produces. The OCL Rule 1.9 enforces the produces relationship to be applied only from produce task to resource. Notice that the ocl IsKindOf operation allows also distribution channels to be produced. This means that a company puts in place a production process that results in a distribution channel for the distribution of some resource.

OCL Rule 1.9: Produces constraint

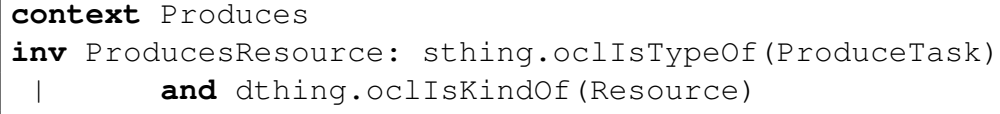

Consumes. The OCL Rule 1.10 enforces the consumes relationship to be applied only from produce task to resource. Notice that the oclis TypeOf operation prohibits to consume distribution channels, for these are not consumable resources (a ticket corner cannot be consumed!). 
OCL Rule 1.10: Consumes constraint

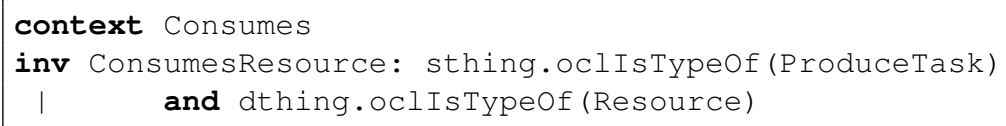

Distributes. The OCL Rule 1.11 enforces the distributes relationship to be applied only from delivery task to resource. Notice that the oclis Typeof operation prohibits to distribute distribution channels.

OCL Rule 1.11: Distributes constraint

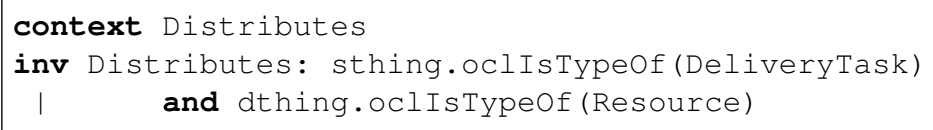

Task (Referenced Part). The OCL Rule 1.12 enforces each task's referenced element to be a task, if specified. The referenced element must be of the same type as the one being evaluated by the rule; the first statement of the rule allows the ref attribute to be undefined.

OCL Rule 1.12: Task (Referenced Part) constraint

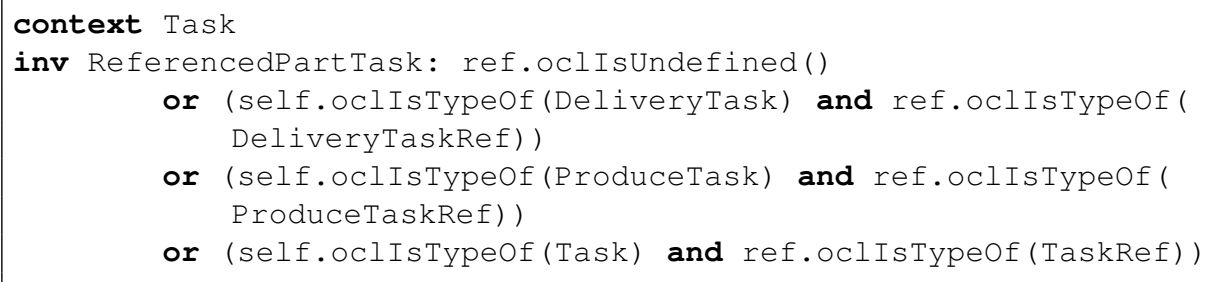

Resource (Referenced Part). The OCL Rule 1.13 enforces each resource's referenced element to be a resource, if specified. The constraint works in the following way:

- a resource can reference only another resource element;

- a distribution channel can reference a distribution channel or a resource element;

The reasons behind this choice is that an actor providing a resource does not necessarily implies such element to be modeled in the same manner by the recipient of such provision. For instance, a construction company may be producing buildings (conceptualized as resource), but the recipient of those can model them as distribution channels (i.e. a shop) through which to deliver its value propositions. Oppositely, an actor supplying a distribution channel implies the provision of an element that is going to be used in that very specific way. 
OCL Rule 1.13: Resource (Referenced Part) constraint

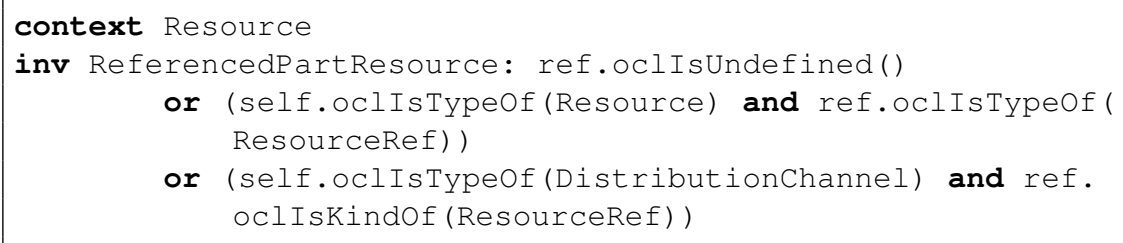

Commitment. The OCL Rule 1.14 defines a constraint to which all the elements within a commitment must apply. Specifically, it enforces each commitmentpart's referenced element to adhere to the following rules:

- a resource can be referenced iff the element is a ResourceRef or a DistributionChannelRef;

- a task can be referenced iff the element is a TaskRef;

- a distribution channel can be referenced iff the element is a DistributionChannelRef;

- a delivery task can be referenced iff the element is a DeliveryTaskRef;

- a produce task can be referenced iff the element is a ProduceTaskRef.

OCL Rule 1.14: Commitment (Referenced Part) constraint

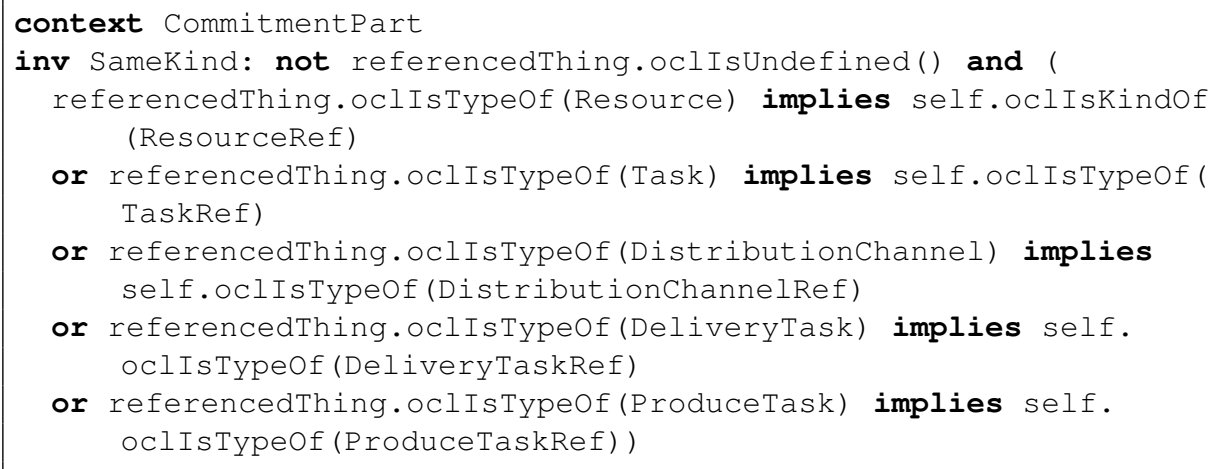

Similarly to OCL Rule 1.12, the commitment over a distribution channel can reference to both a distribution channel and a resource. For instance, a company owning flats and rooms (resources) may providing them, via a commitment, in form of offices opened to public (distribution channels). This way, the debtor is enabled to model the way she is going to provide resources under her ownership and to tailor them according the organization's needs.

\section{From Business Plans to Business Processes}

The Business Process Modeling Notation (BPMN) [16 enables designing business processes as a set of activities (tasks and subprocesses) connected by a control flow. BPMN models can be automatically analyzed and simulated to determine which paths are faster, to detect bottlenecks, to estimate costs, etc. 
BPMN modeling and analysis can be adopted to compare the effectiveness of TBIM tactics. In order to do so, we define of a conceptual mapping between a TBIM model and a set of interconnected BPMN process models. We assume that the indirect tasks and resources within the scope of an actor $A$ appear in the consignment (reward) of at least one commitment made to (by) $A$ by (to) actor $B$, and they appear in the scope of $B$.

Actors. Every agent and role has to appear in at least one process as pool, as lane within a pool, or as additional participant. The same actor can be mapped to different elements and element types. For instance, actor MJF can be mapped to pool MJF Administration and additional participant MJF Vice-President.

Resources. Every direct resource that is produced or consumed by some task has to be mapped to at least one data object. In BPMN, a data object is information about what activities require to be performed and/or what they produce.

Tasks. TBIM tasks - of any type - describe conducted activities in an organization. Every direct task shall be mapped to at least one BPMN task in a BPMN process. These BPMN tasks shall appear in a pool or lane whose performer is the actor that owns the task in the TBIM model.

Resource consumption and production. Produce tasks do produce and consume resources. For direct produce tasks, we require that at least one corresponding BPMN task has incoming and outgoing data association links to data objects that preserve the semantics of consumption and production, respectively.

Delivery. Delivery tasks denote routines for transferring a resource to another actor. For direct delivery tasks, we require the existence of a message flow from the pool/lane corresponding to the actor that owns the delivery task to the pool/lane corresponding to the recipient actor. Distribution channels are mapped indirectly (e.g., via tasks and/or messages), for BPMN has no primitive that carries the semantics of a distribution channel.

Commitments and indirect elements. The guidelines above take into account direct elements (tasks and resources). We examine now indirect elements (gray-colored, which appear in at least one commitment as consignment or reward). We show the case where the indirect element appears in the consignment. The mapping inverts debtor and creditor if the element is in the reward.

- Resources shall be modeled via BPMN message flows between pools or lanes. There should be at least one message from the debtor to the creditor where the message corresponds to the resource.

- Produce tasks shall be modeled as a two-way message flow: the creditor requests the production process, and the debtor provides the process outcome.

- Distribution channels (without delivery task) shall be modeled as a message flow from the debtor to the creditor, where the message is the provided channel (in TBIM, a distribution channel is a resource).

- Delivery tasks shall be modeled as a message from the creditor to the debtor, where the message requests the initiation of the distribution process.

Figure 7 depicts a possible mapping of the Distribute flyers task refinement of Figure 4. In the topmost lane, MJF Administration sends flyers specifications to 
the Ticket Agency company, which provides flyers according to the partnership specifications (Figure 6). After some interactions, the process terminates with a gateway to evaluate two different strategies (subprocesses): relying on an external distribution company, or handling distribution internally.

Our mapping defines compliance criteria between a set of processes and a TBIM model. The analyst can possibly derive process skeletons, but she would typically enrich them with fine-grained information, including additional BPMN tasks, different types of control flow, and structuring in subprocesses.

\section{Evaluating alternative TBIM plans}

Business process simulation enables evaluating processes and the alternatives therein in terms of execution times, usage of resources, and costs 23. Simulation has been used for the analysis of organizations at design-time as well as in realtime environments as strategic and operational decision support tool 2027. We show how, given a set of processes for a TBIM model (Section 5), simulations can be run to gain insights about the quality of alternative TBIM plans. We have used the Adonis BPM toolkit [6] (specifically, its simulation component) to model processes, enrich them, and evaluate alternative plans. In the simulation we present in this section, we use the working environment shown in Figure 8, which structures the actors in the considered organization. We rely on the following assumptions:

- The MJF Administration is the organizational unit dealing with financial, marketing and human resources activities; each performer belonging in this unit is expected to cost $10 \mathrm{units} / \mathrm{h}$ to the organization.

- The Staff members have to deal with several activities: to manage volunteers and recruits, to set up flyers stations and to collaborate in the performing

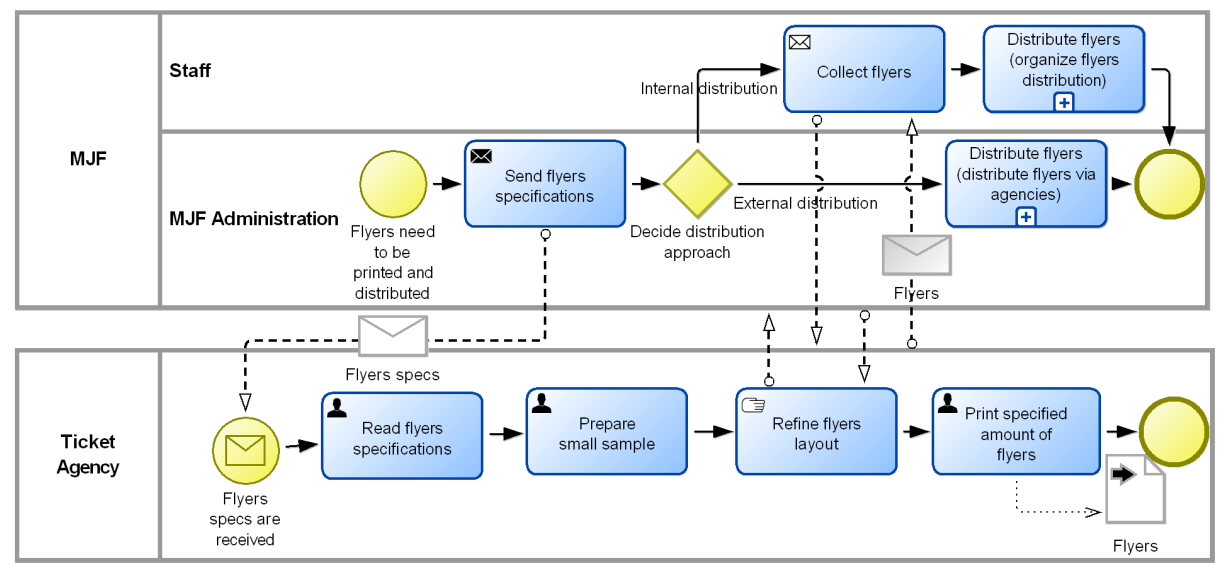

Fig. 7: Overall BPMN model for the distribution of MJF flyers 
of such activity. The hourly wage is set to 20 units/h for the managerial activities and to $10 \mathrm{units} / \mathrm{h}$ for both the remaining two.

- Volunteers are not a cost to the organization as they provide their services free of charge.

- Recruits are recently hired employees with the purpose of delivering flyers to people; each recruit costs 8 units/h.

- Availability determines how much (in percentage) a specific performer is devoted to playing a specific set of roles. For the Distribute flyers role we set the availability value to $50 \%$ for the Volunteers, $30 \%$ for the Staff and $500 \%$ for the Recruits as we simulate the existence of five recruits working full-time on flyers distribution; all the other performers have $100 \%$ availability value.

- The process of distribution is expected to be carried out for 20 business days, continuously.

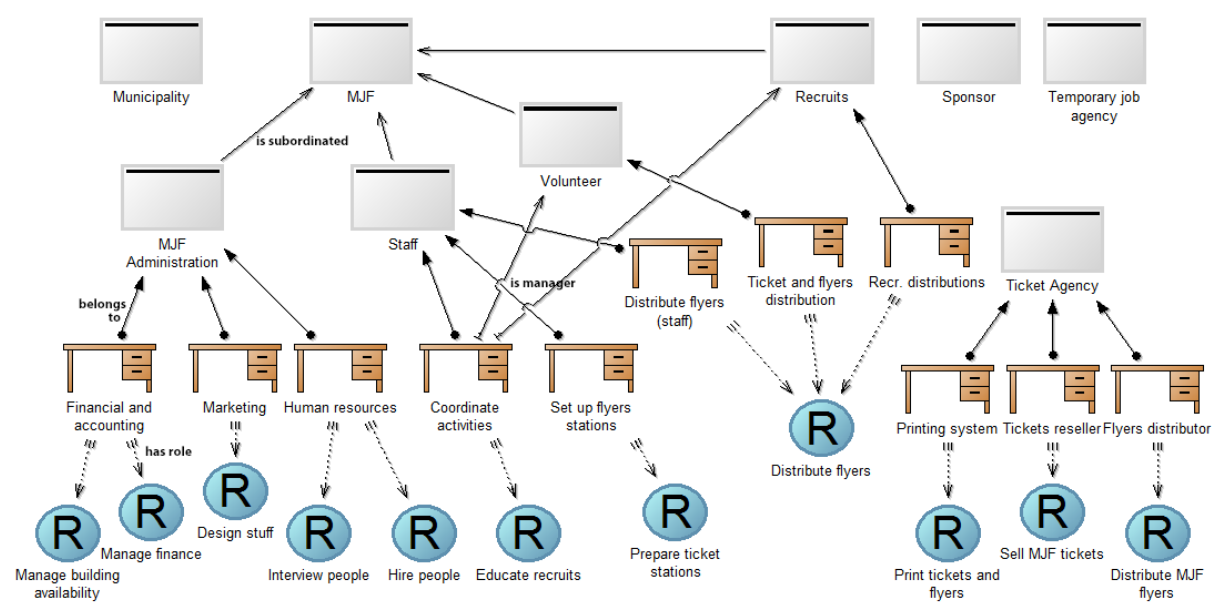

Fig. 8: Partial Mountreux Jazz Festival Working Environment

Given the above, we can now enrich the simulation model with some activityspecific attributes, so to enable the evaluation of alternatives. These attributes concern cost, assigned resources, and expected execution times.

Figure 9 shows the business process for distributing flyers through an external company (Ticket Agency). The process requires MJF Administration to agree on the revenue rate (a TBIM reward). Once agreed, the distribution is taken care of by the ticket agency, with no further involvement of the MJF administration. The simulation configuration model in Figure 10a presents how we modeled this activities flow.

Figure 11 shows a business process for the internal organization of flyers distribution. MJF Administration hires five people among the candidates provided by Temporary job agency. The candidates are interviewed and possibly hired. After 


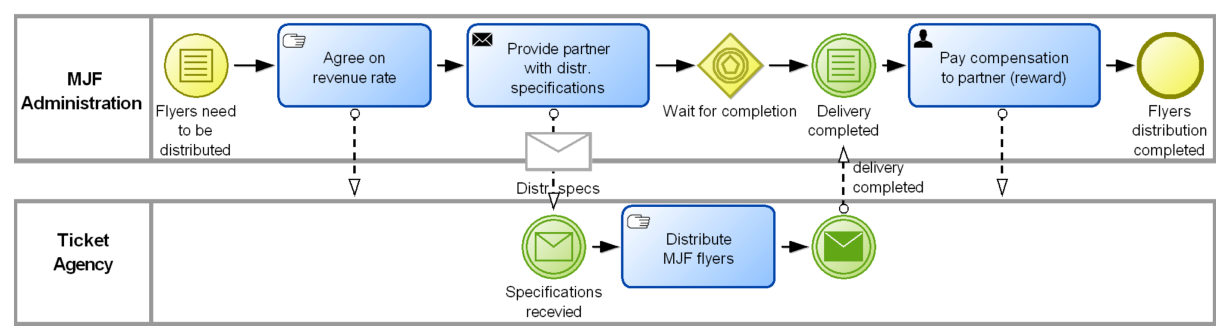

Fig. 9: BPMN model for the external organization of flyers distribution

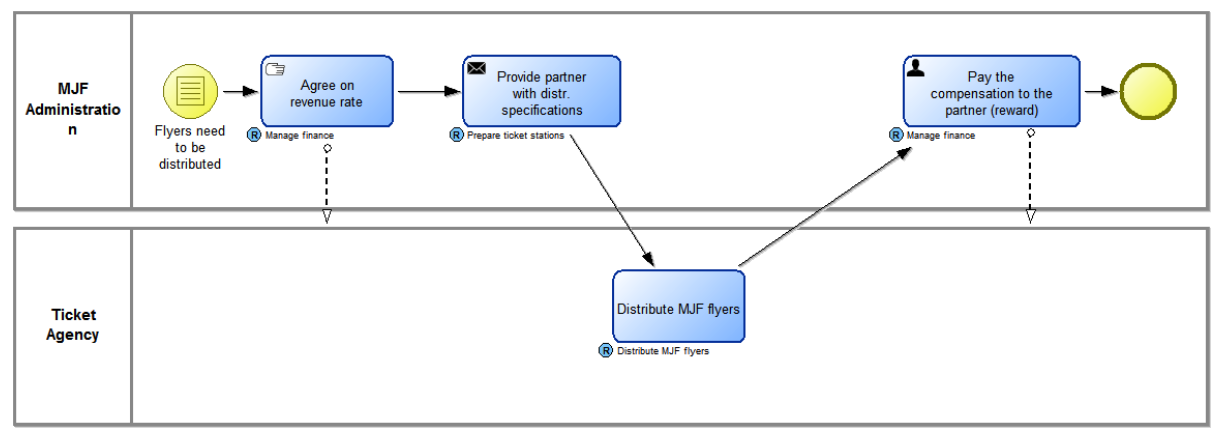

(a) Simulation configuration model

\begin{tabular}{l|r|r|r|r|r} 
& Execution time & Waiting time & Resting time & Transport time & \multicolumn{1}{c}{ Costs } \\
\hline Agree on revenue rate & $00: 000: 02: 00: 00$ & $00: 000: 00: 00: 00$ & $00: 000: 00: 00: 00$ & $00: 000: 00: 00: 00$ & 0,00 \\
\hline Provide partner with distr. specifications & $00: 000: 03: 00: 00$ & $00: 000: 00: 00: 00$ & $00: 000: 00: 00: 00$ & $00: 000: 00: 00: 00$ & 0,00 \\
\hline Pay the compensation to the partner (reward) & $00: 000: 00: 30: 00$ & $00: 000: 00: 00: 00$ & $00: 000: 00: 00: 00$ & $00: 000: 00: 00: 00$ & 500,00 \\
\hline Distribute MJF flyers & $00: 020: 00: 00: 00$ & $00: 000: 00: 00: 00$ & $00: 000: 00: 00: 00$ & $00: 000: 00: 00: 00$ & 0,00
\end{tabular}

(b) Simulation configuration parameters

Fig. 10: Simulation configurations for MJF external flyers distribution

a training period, the ticket corners are set up and provided with flyers, and the distribution starts. MJF Administration needs to cope with personnel sick leaves or resignations. From the simulation's perspective, we modeled these interactions as shown in Figure 12a. In order to simulate the plausible scenario of one or more recruits to resign from the job (or to get sick, or fired because negligent), we execute such process in parallel with a gateway that determines whether everything went smooth during the distribution process. This probability, which is set to have troubles with recruits in the $30 \%$ of the cases, allows us to model the scenario in which the company needs to find a substitute for those whom are going to be asked to leave MJF services. A uniformly distributed probability 


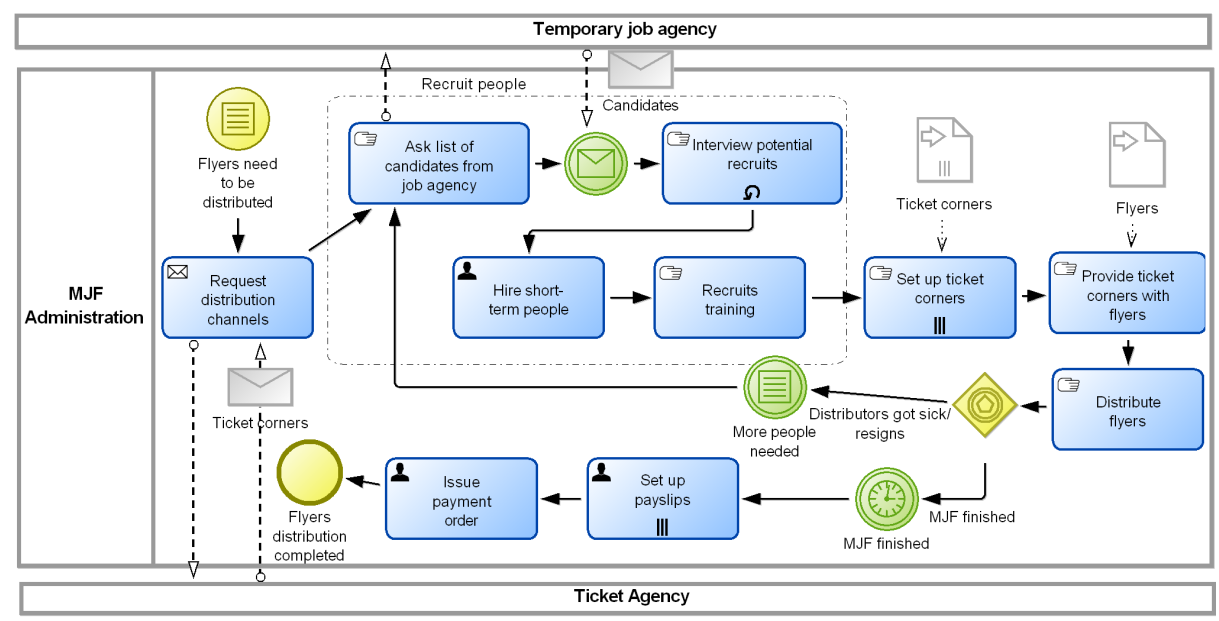

Fig. 11: BPMN model for the internal organization of flyers distribution

will draw a number in the range $[1,3]$ of such people. The recruit interactions shall start once more until the number of five has been reached again.

Interpreting the results. The outcome of the simulation is computed using 100,000 simulation runs. The results from process simulations include overall process costs, time spent in cycles, frequencies for each task, etc.

Figure 13 presents the comparison between time needed to distribute flyers using the two different approaches. As expected, the two values are close to 20 days, as the distribution process has a remarkable impact in the overall execution times. Still, the majority of time spent in the processes is attributed to Ticket Agency and Recruits, respectively in the external and internal scenarios. In the external scenario, the agency has to deal with everything related to the distribution process: preparation of the ticket stations, training of the recruits team, and the actual distribution of flyers to people. In the internal scenario, the recruits have to deal with the distribution but the preparations (recruiting and training them and setting up the stations with flyers) are up to other roles within the organization. Moreover, Staff members and Volunteers are expected to contribute in this activity. According to the simulations, the internal scenario takes longer, for the preparations must be done by the organization itself. In particular, the time spent recruiting and educating people is the $7 \%$ of the overall process execution times, as shown in Figure 14.

From the cost perspective, we distinguish between personnel and management costs. The former are summarized in Figure 15, while the latter are shown in Figure 16. Our assumptions imply that there exists a significant gap between personnel costs in the two different scenarios. By delegating the distribution to an external partner, both the Staff and the MJF Administration organizational units would be relieved from some effort as the activities are entirely carried out by the Ticket Agency partner. In the internal scenario, instead, distribution 


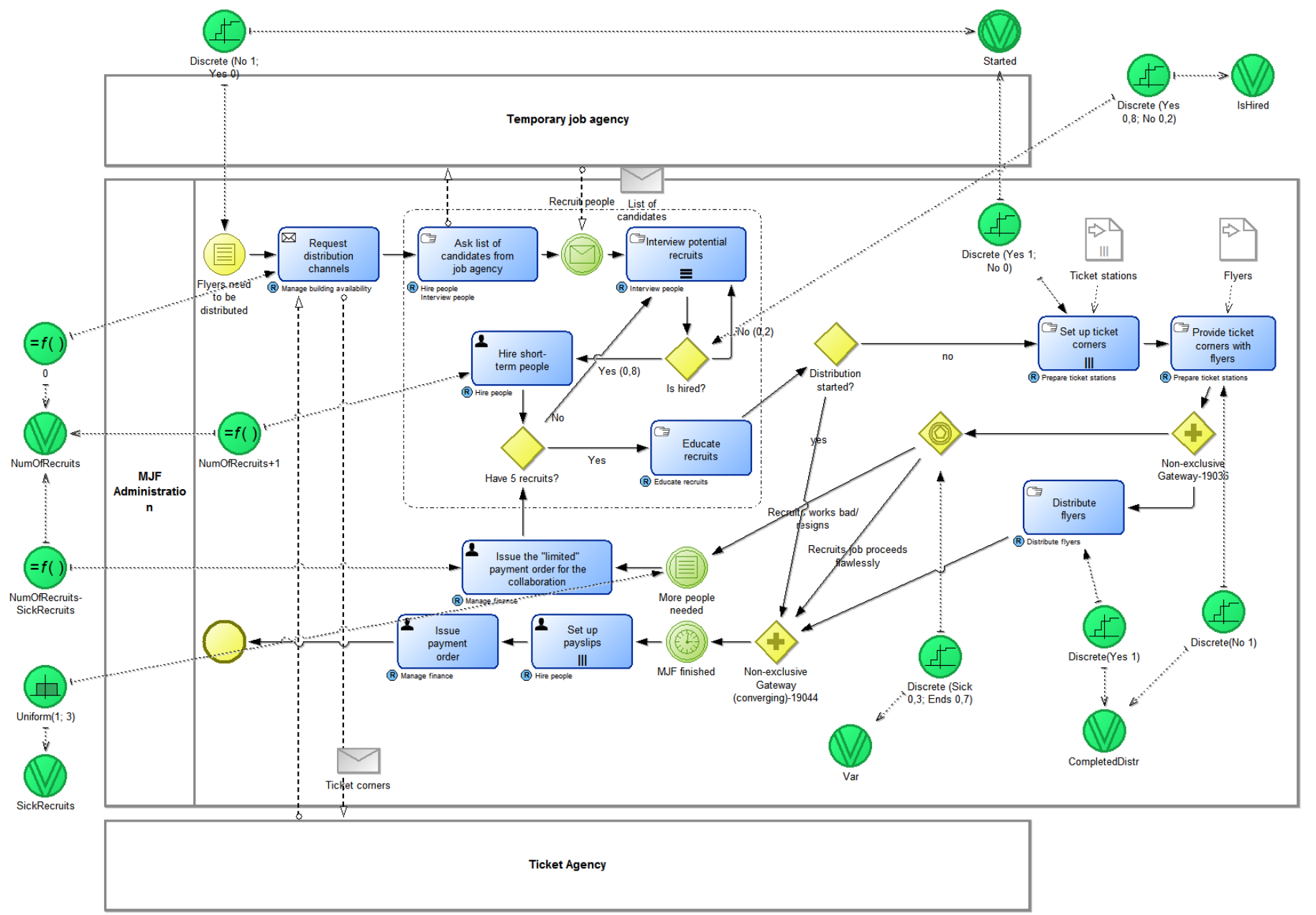

(a) Simulation configuration model

\begin{tabular}{|l|r|r|r|r|r|} 
& Execution time & Waiting time & Resting time & Transport time & \multicolumn{1}{c|}{ Costs } \\
\hline Request distribution channels & $00: 000: 03: 00: 00$ & $00: 002: 00: 00: 00$ & $00: 000: 00: 00: 00$ & $00: 000: 00: 00: 00$ & 0,00 \\
\hline Ask list of candidates from job agency & $00: 000: 00: 10: 00$ & $00: 000: 00: 00: 00$ & $00: 000: 00: 00: 00$ & $00: 000: 00: 00: 00$ & 100,00 \\
\hline Interview potential recruits & & & & & \\
\hline Hire short-term people & $00: 000: 01: 00: 00$ & $00: 000: 00: 00: 00$ & $00: 000: 00: 20: 00$ & $00: 000: 00: 00: 00$ & 10,00 \\
\hline Educate recruits & $00: 000: 00: 20: 00$ & $00: 000: 00: 00: 00$ & $00: 000: 00: 00: 00$ & $00: 000: 00: 00: 00$ & 0,00 \\
\hline Set up ticket corners & $00: 000: 01: 30: 00$ & $00: 000: 00: 00: 00$ & $00: 000: 00: 00: 00$ & $00: 000: 00: 00: 00$ & 0,00 \\
\hline Provide ticket corners with flyers & $00: 000: 02: 00: 00$ & $00: 000: 00: 00: 00$ & $00: 000: 00: 00: 00$ & $00: 000: 00: 00: 00$ & 50,00 \\
\hline Distribute flyers & $00: 000: 00: 00: 00$ & $00: 000: 00: 00: 00$ & $00: 000: 00: 00: 00$ & $00: 002: 00: 00: 00$ & 50,00 \\
\hline Set up payslips & $00: 020: 00: 00: 00$ & $00: 000: 00: 00: 00$ & $00: 000: 00: 00: 00$ & $00: 000: 00: 00: 00$ & 0,00 \\
\hline Issue payment order & $00: 000: 01: 00: 00$ & $00: 000: 00: 00: 00$ & $00: 000: 00: 00: 00$ & $00: 000: 00: 00: 00$ & 0,00 \\
\hline Issue the "limited" payment order for the collaboration & $00: 000: 00: 10: 00$ & $00: 000: 00: 00: 00$ & $00: 000: 00: 00: 00$ & $00: 000: 00: 00: 00$ & $0,00: 00: 30: 00$ \\
\hline
\end{tabular}

(b) Simulation configuration parameters

Fig. 12: Simulation configuration for MJF internal flyers distribution 
requires the allocation of Staff members and Volunteers. Our simulation relies on the hypothetical scenario in which Staff members and Volunteers are not sufficient to take care of flyers distribution on their own: therefore, the MJF Administration is asked to hire people to carry out that specific activity. These Recruits have a noticeable impact on personnel costs.

The management costs outcome is orthogonal to the personnel costs. In the simulation, the costs needed to maintain the network of partnerships is much lower in the internal scenario, for the MJF organization is carrying out most of the activities. The cost associated with the flyers distribution partner, 500 in our settings, is the discriminating factor: the higher the value, the less affordable the strategy becomes.

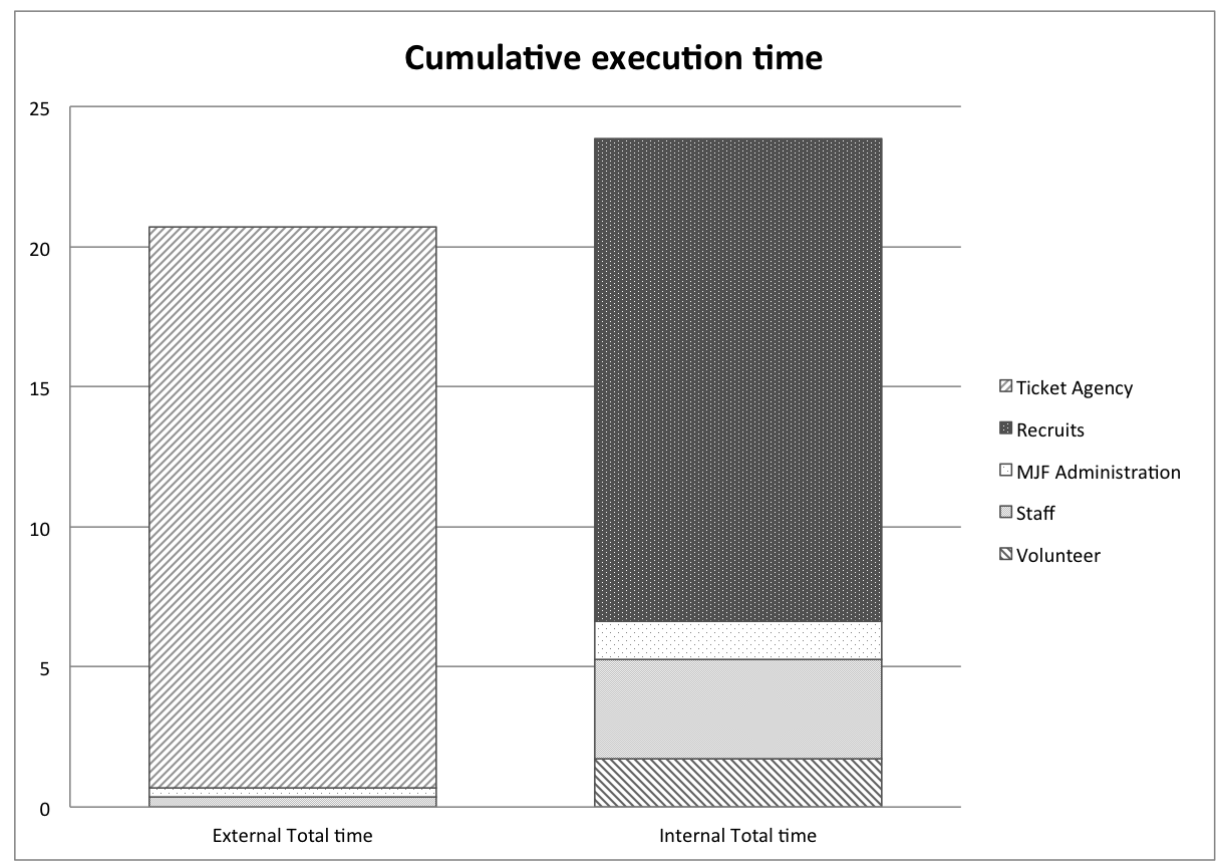

Fig. 13: Role-based cumulative execution times

The aforementioned results are summarized in Table 1. The outcome evidences that the internal approach is expected to take longer than the external. This is due to set-up activities that shall precede flyers distribution. More importantly, the internal process is significantly more expensive in terms of personnel and other costs. Also, both Staff and MJF Administration would be relieved from some effort by relying on a partnership.

Another interesting evaluation parameter is the cycle time. It represents the time needed to completely carry out all the activities and, besides including execution times, it also includes waiting, transport and resting times. The cycle 


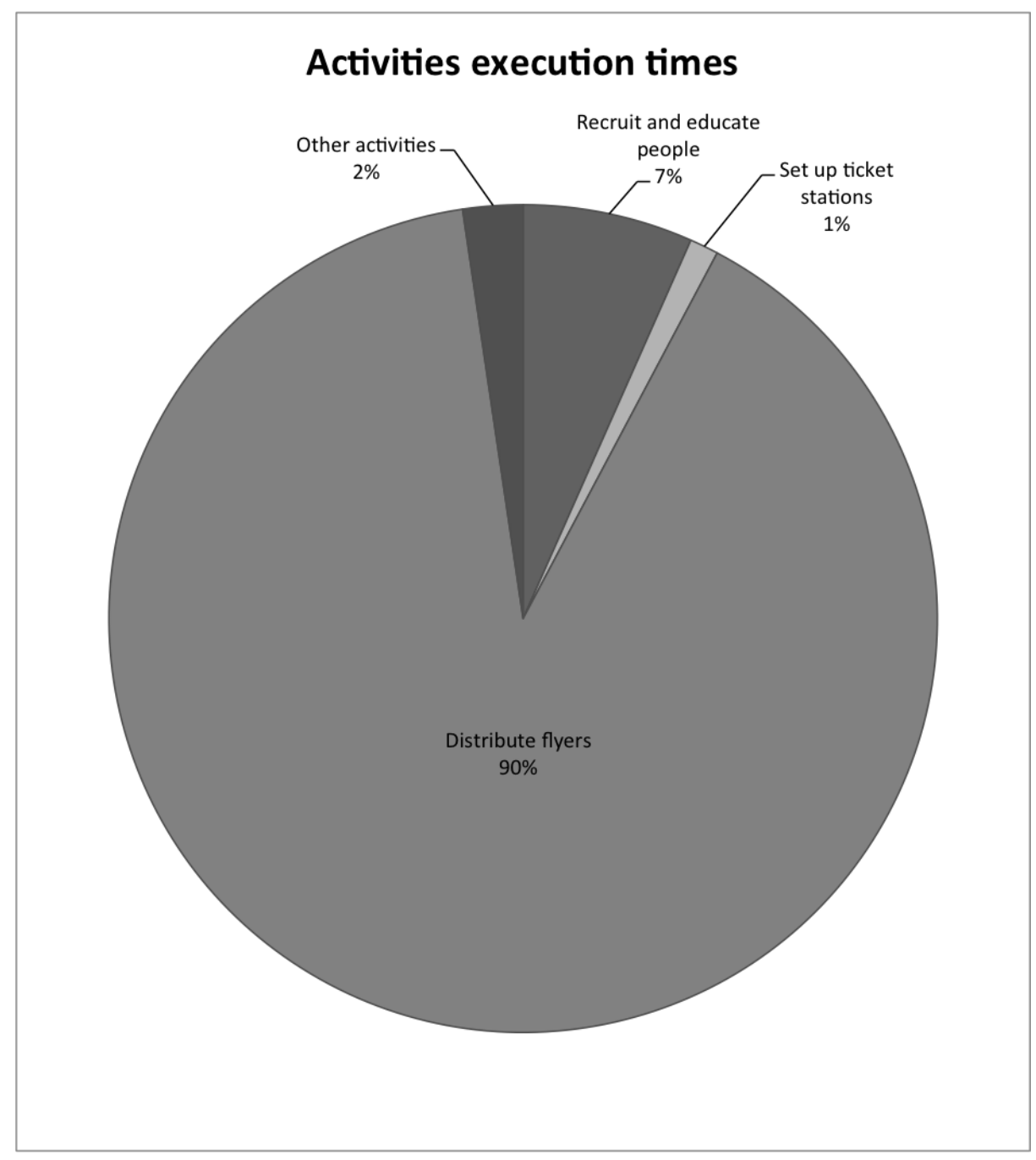

Fig. 14: Per activity execution times 


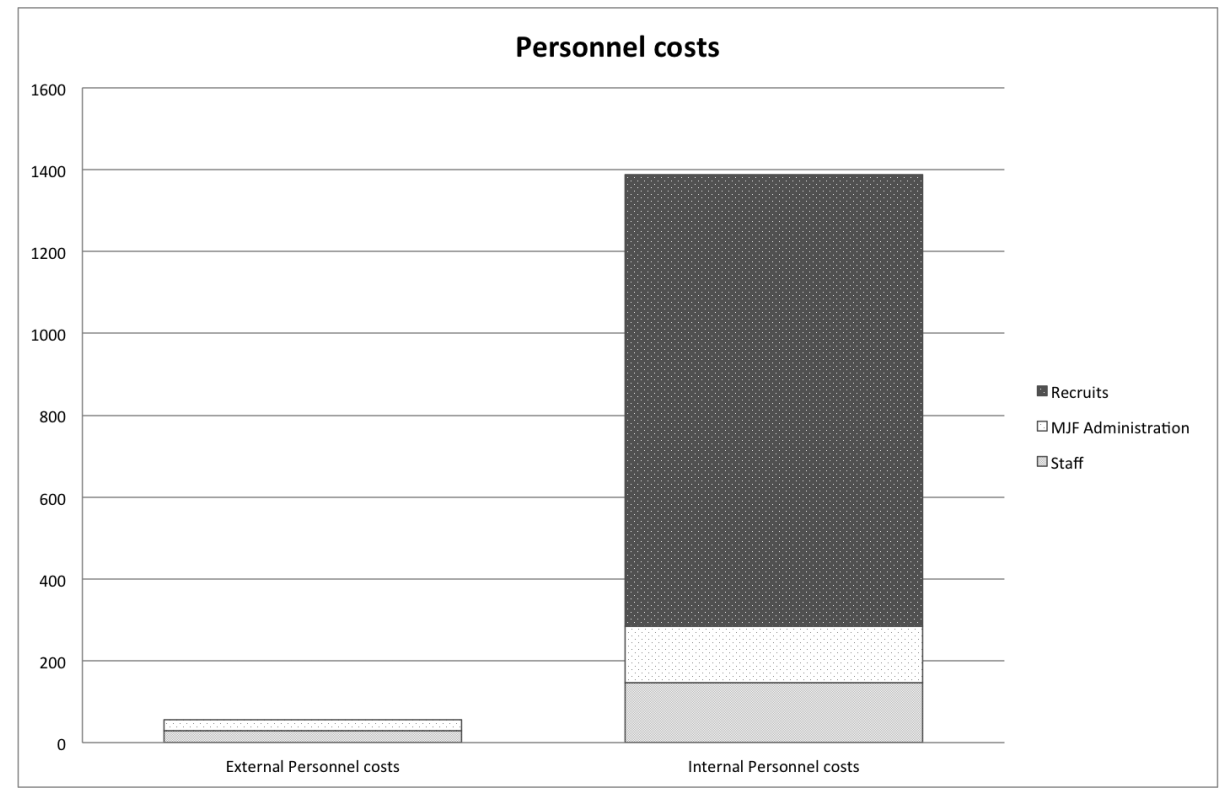

Fig. 15: Organizational unit-based personnel costs

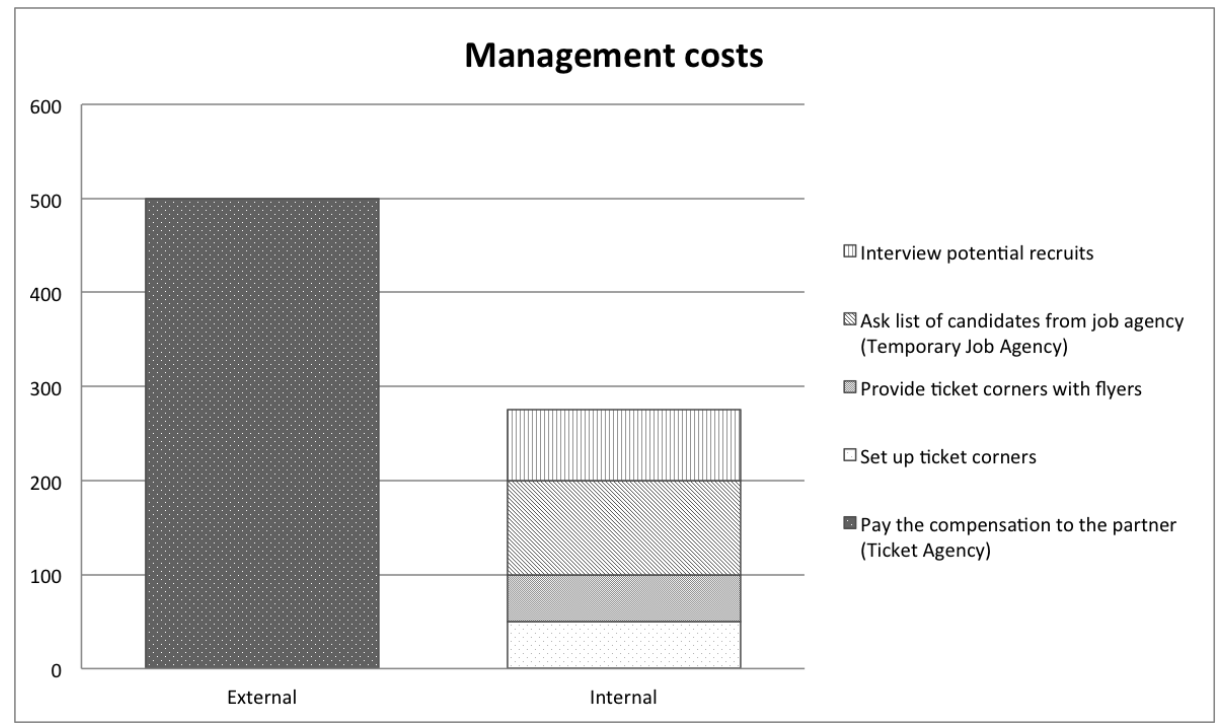

Fig. 16: Activity-based other costs 
Table 1: Simulation results for the MJF flyers distribution process. Times are in business days, costs are in units

\begin{tabular}{l|c|r|r|r|r|c}
\multirow{2}{*}{ Organizational Unit } & \multicolumn{2}{|c|}{ Time } & \multicolumn{2}{c}{ Pers. Costs } & \multicolumn{2}{c}{ Mgmt. Costs } \\
\cline { 2 - 7 } & Ext. & \multicolumn{1}{|c|}{ Int. } & Ext. & Int. & Ext. & Int. \\
\hline Volunteer & 0.0 & 1.71 & 0.0 & 0.0 & 0.0 & 0.0 \\
Staff & 0.37 & 3.55 & 30.0 & 145.71 & 0.0 & 100.0 \\
MJF Administration & 0.31 & 1.35 & 25.0 & 138.42 & 500.0 & 175.07 \\
Recruits & 0.0 & 17.25 & 0.0 & 1104.0 & 0.0 & 0.0 \\
Ticket Agency & 20.0 & \multicolumn{1}{|c|}{0.0} & & & & \\
\hline Total & 20.68 & 23.86 & 55.0 & 1388.13 & 500.0 & 275.07
\end{tabular}

time for the external approach is 20.69 days, while the internal is 26.23 days. In the external scenario, the MJF organization is capable of arranging the distribution process in roughly a business day as the Ticket Agency company deals with the rest. In the internal scenario, the MJF organization has to deal with many activities prior to distributing flyers. These activities are expected to take more than six business days to complete.

Improving TBIM models. The simulation results can be used to ameliorate the TBIM tactic and/or to choose among alternatives (e.g., different tasks in an OR-refinement, or alternative partnerships). This activity relies on the expertise of the analysts. In our example, the results suggest to keep the partnership with a Ticket agency (Figure 6), and to choose delivery task Distribute flyers via agencies over Organize flyers distribution (Figure 4), thereby making channel Ticket corner useless.

\section{Related work}

We review related work about modeling different aspects of enterprises.

Business ontologies. They define concepts to conceive enterprises. Two key approaches are Uschold's enterprise ontology [24] and the Resource/Event/Agent generalized accounting model [15. The Business Motivation Model [17] defines business plans by starting from the motivations of a company. These works provide sets of concepts (e.g., resources, duality, agents, strategy, activities, motivations) that are at the basis of several modeling languages, including TBIM.

Enterprise architectures. They provide principles, methods, and models for the design and realization of an enterprise. TOGAF [1] promotes a requirementscentered design of the architecture, which begins with a vision of the architecture, and includes governance and change management. The Zachman framework 29] models enterprises by filling all the cells in a matrix where rows define the granu- 
larity level, and columns specify different aspects (why, when, what, how, where, who). These approaches do not offer a specific modeling language.

Business modeling languages. They represent different aspects of a business. The $\mathrm{e}^{3}$ value 9 methodology models a network of enterprises creating, distributing, and consuming resources having an economic value. As observed by Andersson et al. 2, some concepts in BMO are similar to those of $\mathrm{e}^{3}$ value . Lo and $\mathrm{Yu}$ [14] suggest the usage of extended $i^{*}$ [28] agent- and goal-oriented models to design collaborations - including resource exchange and task execution-among organizations. TBIM brings this notion further by suggesting different types of tasks (production, distribution), and uses commitments for relating business partners. $i^{*}$ and $\mathrm{e}^{3}$ value have been combined [10] to support e-service design. In their approach, the gaps between two models are filled in by the analyst. TBIM, instead, relies on a unified conceptual model.

Social commitments. They are relationships that tie together autonomous participants through declarative contracts 21]. Telang et al. 22] rely on commitments to propose an agent-oriented approach for specifying and verifying cross-organization business models. TBIM relies on a more fine-grained ontology for both intentional elements and commitments.

Business Process Modeling (BPM). This activity is intended to create models that represent the business processes within an organization, which are a major component of an enterprise's tactic. BPMN [26] is the de-facto standard notation for BPM, and relies on the notions of activity and control flow. BPMN 2.0 [16] introduce support for the collaboration between different organizations through the collaboration and choreography diagrams. We use business process models to analyze and evaluate alternative tactics. Our future work includes investigating the effectiveness of alternative BPM languages.

\section{Discussion and future work}

We have proposed graphical syntax and semantics of TBIM, a conceptual modeling language that enables modeling and analysis for business plans. TBIM builds on the BIM language, and extends it with primitives derived from the BMO ebusiness ontology. We have also provided guidelines to map TBIM tactics to BPMN processes, and shown how business process simulation techniques can be used to improve and choose among alternative TBIM tactics.

A key feature of TBIM is to decouple the internal tactics of an enterprise (tactical view) from the partnerships with other enterprises and customers (partnership view). This distinction enables determining if there exist unneeded partners, and if some tactical choice is not supported by any partnership.

Evaluation. We have illustrated TBIM and the usage of business process simulations with snippets from the MJF case study. Extensive models are available 
in Francesconi's dissertation [8] (the graphical syntax is slightly different, while the semantics is unvaried).

Implementation. We have developed a proof-of-concept modeling tool to support the TBIM graphical notation. The tool is built using the meta-modeling development platform ADOxx [7]. This choice aims to facilitate integration with the Adonis BPM toolkit, which supports BPMN modeling and features sophisticated analysis and simulation algorithms. Our tool uses the ADOscript programming language for checking consistency and validity of created models.

Future work includes (i) improving the modeling tool to enable public use; (ii) developing features for automatically generating BPMN skeleton processes from TBIM models; and (iii) evaluating TBIM on industrial case studies.

\section{References}

1. D. F. Abell. Defining the business: the starting point of strategic planning. Prentice Hall, 1980.

2. B. Andersson, M. Bergholtz, A. Edirisuriya, T. Ilayperuma, P. Johannesson, J. Gordijn, et al. Towards a reference ontology for business models. In Proc. of ER, pages 482-496. 2006.

3. S. Bagchi and B. Tulskie. E-business models: integrating learning from strategy development experiences and empirical research. In Proc. of the SMS Annual International Conference, pages 15-18, 2000.

4. T. R. Dealtry. Dynamic SWOT analysis: developer's guide. Intellectual Partnerships, 1992.

5. U. Essler and R. Whitaker. Re-thinking e-commerce business modelling in terms of interactivity. Electronic Markets, 11(1):10-16, 2001.

6. BOC Europe. Adonis BPM Toolkit. http://www.boc-eu.com/

7. BOC Europe. ADOxx platform. http://www.adoxx.org/

8. F. Francesconi. Modelling business plans with TBIM: a language for strategic planning. Master's thesis, University of Trento, 2012.

9. J. Gordijn, H. Akkermans, and J. Van Vliet. Designing and evaluating e-business models. IEEE Intelligent Systems, 16(4):11-17, 2001.

10. J. Gordijn, E. Yu, and B. van der Raadt. E-service design using i* and e/sup 3/value modeling. IEEE Software, 23(3):26-33, 2006.

11. The Open Group. TOGAF Version 9. The Open Group Architecture Framework. 2009.

12. J. Horkoff, A. Borgida, J. Mylopoulos, D. Barone, L. Jiang, E. Yu, and D. Amyot. Making data meaningful: The business intelligence model and its formal semantics in description logics. In Proc. of ODBASE, pages 700-717. 2012.

13. R. S. Kaplan and D. P. Norton. Having trouble with your strategy?: Then map it. Harvard Business School Publishing Corporation, 2000.

14. A. Lo and E. Yu. From business models to service-oriented design: a reference catalog approach. In Proc. of ER, pages 87-101, 2007.

15. W. E. McCarthy. Data environment. The Accounting Review, 57(3):554-578, 1982.

16. OMG. Business Process Modeling Notation (BPMN) version 2.0. Technical report, 2006.

17. OMG. Business Motivation Model Specification v1.1. Technical report, 2010. 
18. OMG. Object Management Group Object Constraint Language (OCL). formal/2012-05-09, April 2012.

19. A. Osterwalder. The Business Model Ontology. PhD thesis, HEC Lausanne, 2004.

20. H. A. Reijers and W. M. P. Van der Aalst. Short-term simulation: bridging the gap between operational control and strategic decision making. In Proc. of IASTED $M S$, pages 417-421, 1999.

21. M. P Singh. An ontology for commitments in multiagent systems: Toward a unification of normative concepts. Artificial Intelligence and Law, 7:97-113.

22. P. R. Telang and M. P. Singh. Specifying and verifying cross-organizational business models: An agent-oriented approach. IEEE Transactions on Services Computing, 5(3):305-318, 2012.

23. K. Tumay. Business process simulation. In Proc. of the Winter Simulation Conference, pages 55-60. IEEE, 1995.

24. M. Uschold, M. King, S. Moralee, and Y. Zorgios. The enterprise ontology. The Knowledge Engineering Review, 13(1):31-89, 1998.

25. B. Wernerfelt. A resource-based view of the firm. Strategic management journal, $5(2): 171-180,1984$.

26. S. A. White and D. Miers. BPMN modeling and reference guide. Future Strategies Inc., 2008.

27. M. T. Wynn, M. Dumas, C. J. Fidge, A. H. M. Hofstede, and W. M. P. Aalst. Business process simulation for operational decision support. In Proc. of BPM Workshops, pages 66-77. 2008.

28. E. Yu. Modelling Strategic Relationships for Process Reengineering. PhD thesis, University of Toronto, 1996.

29. J. A. Zachman. A framework for information systems architecture. IBM systems journal, 26(3):276-292, 1987. 